\title{
Sonological and Pathological Evaluation of Solitary Nodule Thyroid
}

\author{
Dr Abid Ali, Dr Mohammed Jasir \\ Associate Professor, Department Of General Surgery; Mes Medical College; Perintalmanna;India \\ Department Of General Surgery ;Mes Medical College; Perintalmanna;India
}

\begin{abstract}
Thyroid nodules are a common clinical problem. Epidemiologic studies have shown the prevalence of palpable thyroid nodules to be approximately $5 \%$ in women and $1 \%$ in men living in iodine sufficient parts of world ${ }^{[1]}$

Most of the thyroid nodules are benign and only less than $7 \%$ of thyroid nodules are malignant ${ }^{[2]}$. So it is very important to differentiate between benign and malignant condition preoperatively

So the goal of diagnostic workup is to select those patients for surgery who have a high likelihood of harbouring malignancy in nodule. Many procedures are used in diagnostic workup of solitary nodule of thyroid- ie TFT, FNAC, USG

FNAC is considered as the most accurate and cost effective method for evaluating thyroid nodule. The sensitivity and accuracy of FNAC is as high as $95 \%$ in experienced hands. Positive predictive value of 90-98\% and negative predictive value of 94-99\% established FNAC as a valuable diagnostic modality ${ }^{[3]}$. Although in our settings we usually found that FNAC results are usually not confirmatory

Ultrasound is considered as the imaging modality of choice for investigation of thyroid nodules.According to new ATA guidelines (AMERICAN THYROID ASSOCIATION) diagnostic ultrasound should be performed in all patients with a suspected thyroid nodule ${ }^{[4]}$.Sensitivity of ultrasound has been demonstrated as high as $86.5 \%$ and specificity as high as $92.3 \%{ }^{[5]}$

The purpose of this study is to know the efficiency of ultrasound in evaluating solitary nodule of thyroid. It compares final histopathology and ultra sound findings to know the significance of ultrasound in evaluating solitary thyroid nodule
\end{abstract}

Keywords: fine needle aspiration cytology, malignancy, Solitary nodule thyroid, ultrasound thyroid.

\section{Introduction}

Thyroid nodules are a common clinical problem. Epidemiologic studies have shown the prevalence of palpable thyroid nodules to be approximately $5 \%$ in women and $1 \%$ in men living in iodine sufficient parts of world $^{[1]}$.

Most of the thyroid nodules are benign and only less than $7 \%$ of thyroid nodules are malignant ${ }^{[2]}$. So it is very important to differentiate between benign and malignant condition preoperatively

So the goal of diagnostic workup is to select those patients for surgery who have a high likelihood of harbouring malignancy in nodule. Many procedures are used in diagnostic workup of solitary nodule of thyroidie TFT, FNAC, USG

FNAC is considered as the most accurate and cost effective method for evaluating thyroid nodule. The sensitivity and accuracy of FNAC is as high as $95 \%$ in experienced hands. Positive predictive value of $90-98 \%$ and negative predictive value of $94-99 \%$ established FNAC as a valuable diagnostic modality ${ }^{[3]}$. Although in our settings we usually found that FNAC results are usually not confirmatory

Ultrasound is considered as the imaging modality of choice for investigation of thyroid nodules.According to new ATA guidelines (AMERICAN THYROID ASSOCIATION) diagnostic ultrasound should be performed in all patients with a suspected thyroid nodule ${ }^{[4]}$.Sensitivity of ultrasound has been demonstrated as high as $86.5 \%$ and specificity as high as $92.3 \%{ }^{[5]}$

The purpose of this study is to know the efficiency of ultrasound in evaluating solitary nodule of thyroid. It compares final histopathology and ultra sound findings to know the significance of ultrasound in evaluating solitary thyroid nodule

\section{Aim Of Study}

1. To know the efficiency of ultrasound in determining the malignancy among solitary nodule thyroid.

2.To know how many cases of solitary nodule are diagnosed to be having malignancy after final histopathology report. 


\section{Materials And Methods}

Present study includes a total number of 80 patients with solitary nodule thyroid who underwent thyroidectomy in general surgery department of MES medical college and followed up for a period of 2 years from October 2011-October 2013.

A detailed history and clinical examination of all patients were done. Routine investigations ,thyroid function test and ENT examination including indirect laryngoscopy were done for all.

In all patients FNAC was done. It required a $10 \mathrm{cc}$ syringe, a $22 \mathrm{G}$ needle and 3 slides $(75 \mathrm{X} 25 \mathrm{~mm})$.This was done as an outpatient procedure .Patient lay supine on a table with a pillow beneath the shoulders to keep the neck extended and the anterior neck muscles relaxed. The gland was fixed in between the fingers and thumb. The overlying skin was cleansed with antiseptic . No local anaesthesia was given. The lesion was punctured with a 22 gauge needle. When the needle entered the nodule the needle was moved back and forth with in the nodule till enough material was aspirated in the hub of the needle. The needle was taken out of the nodule.Gentle pressure was applied to control bleeding. Some air was sucked into the syringe .The needle was now attached to the syringe and material in hub of needle was expelled onto a slide. With gentle pressure with another slide a thin smear was made. The procedure was repeated. Smears was prepared by air drying or fixing in equal amounts of absolute alcohol and ether for 15 minutes which was kept in a koplins jar. Air dried smears were stained by geimsa stain and alcohol fixed smear were stained by papinicoloau stain. Slides were viewed under low power and high power microscope for evidence of malignancy.

ultrasonogram neck was done in all patients.they were examined in supine position with neck extended and small pad placed transversely under the shoulder to provide better exposure of neck. The thyroid gland was then examined in both transverse and longitudinal planes. imaging of lower pole was enhanced by asking the patient to swallow which momentarily raised the thyroid gland in the neck. The region near carotid vessel was looked for any enlarged lymphnodes

Surgery was done in all patients. The surgical procedure were total, subtotal, hemi Thyroidectomy. If nodes were involved they were removed by modified or selective neck dissection

Specimens were sent for histopathological examinations. They were fixed by keeping them in $10 \%$ formalin about 10 times the volume of tissue. They were sent to pathologist after labeling and with a proper request. In the department of pathology they were numbered. after 24 hours bits were taken from specimens and they were processed with dehydration in ascending grades of alcohol. Blocks were prepared by waxing the bits. Thin sections were taken and stained with haematoxylin and eosin. Slides were viewed under low power and high power microscope and histopathological reports were made

These patients were followed up during the immediate postoperative period for the presence of complications like vocal cord paralysis and hypoparathyroidism.

\section{Figures And Tables}

Table 1

\begin{tabular}{|l|c|}
\hline Period of study & october 2011-october2013 \\
\hline No. of cases studied & 80 \\
\hline No. of cases with FNAC & 80 \\
\hline No. of cases with USG neck & 80 \\
\hline
\end{tabular}

Fig: 1

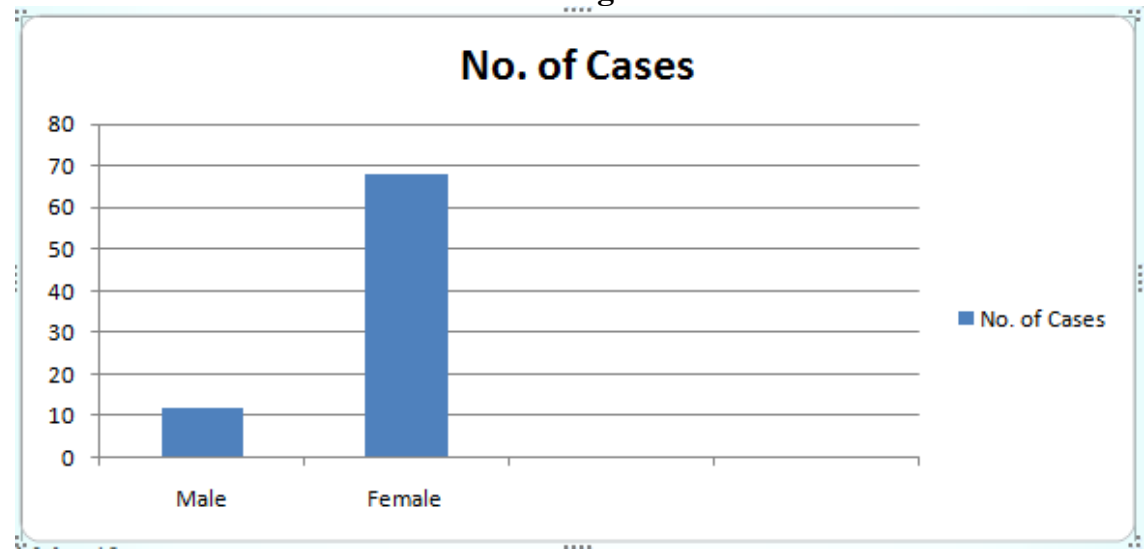

Males- 12

Females-68 
TABLE 2

BENIGN DISEASES

\begin{tabular}{|l|c|c|c|c|}
\hline \multirow{2}{*}{$\begin{array}{l}\text { Histo } \\
\text { logical type }\end{array}$} & \multicolumn{2}{|c|}{$\begin{array}{l}\text { Sex } \\
\text { No of cases }\end{array}$} & \multicolumn{2}{|c|}{ Total } \\
\cline { 2 - 5 } & male & female & No of cases & \\
\hline Colloid nodule & & 28 & 34 & 58.62 \\
\hline Thyroiditis & 6 & 19 & 19 & 32.75 \\
\hline Adenoma & 0 & 5 & 5 & 8.62 \\
\hline
\end{tabular}

Fig:2

\section{Benign Diseases}

\section{INFERENCE}

Among the benign disorders, commonest was nodular goiter $(58.62 \%)$, followed by thyroiditis $(32.75 \%)$

$>$ Thyroiditis \& adenoma was seen in females.

$>$ For colloid nodule, male:female ratio was 1:4.67

TABLE 3 :

Malignant Diseases

\begin{tabular}{|c|c|c|c|c|}
\hline \multirow[t]{3}{*}{ Histological Type } & Sex & & \multirow[t]{2}{*}{ Total } & \\
\hline & \multicolumn{2}{|c|}{ No. of cases } & & \\
\hline & Males & Females & No. of cases & $\%$ \\
\hline Papillary Carcinoma Thyroid & 4 & 11 & 15 & 68.1 \\
\hline Follicular carcinoma Thyroid & 2 & 5 & 7 & 31.8 \\
\hline Others & 0 & 0 & 0 & 0 \\
\hline
\end{tabular}

Fig:3

\section{Malignant Diseases}

\section{INFERENCE}

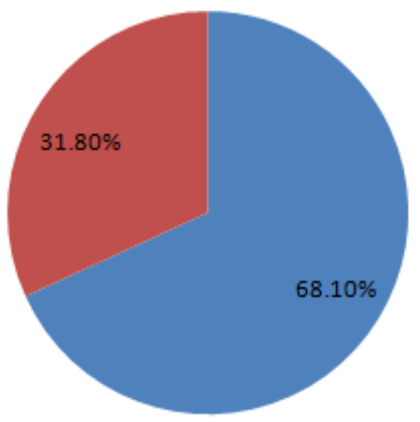

Colloid nodule

- Thyroiditis

adenoma

$\rightarrow$ Among the malignant diseases, papillary carcinoma thyroid was the commonest $(68.1 \%)$ followed by follicular carcinoma thyroid $(38.1 \%)$

$\rightarrow$ For thyroid malignancies, male:female ratio was 1.5:4

$\rightarrow$ Ratio of malignant : benign diseases in the study was $1: 7.3$ 
TABLE 4

AGE \& SEX DISTRIBUTION FOR MALIGNANCY

\begin{tabular}{|l|c|c|c|c|}
\hline \multirow{2}{*}{ Age in years } & \multicolumn{2}{|c|}{ Males } & \multicolumn{2}{c|}{ Females } \\
\cline { 2 - 5 } & Benign & Malignant & Benign & 0 \\
\hline $0-20$ & 0 & 0 & 2 & 6 \\
\hline $21-30$ & 3 & 0 & 22 & 5 \\
\hline $31-40$ & 6 & 0 & 11 & 4 \\
\hline $41-50$ & 2 & 4 & 0 & 2 \\
\hline $51-60$ & 0 & 0 & 2 & 1 \\
\hline $61-70$ & 0 & 0 & 0 & 0 \\
\hline$>70$ & 0 & 4 & 52 & 18 \\
\hline Total & 11 & & & 0 \\
\hline
\end{tabular}

\section{INFERENCE}

$\rightarrow$ Of the total malignant cases, 4 (18.1\%) were males

$\rightarrow$ Of the total malignant cases, $18(81.2 \%)$ were females

$\rightarrow$ Proportion of malignancy in male was $26.6 \%$

$\rightarrow$ Proportion of malignancy in females were $25.7 \%$

$\rightarrow$ Proportion of malignancy in 30-60 age group was $27.1 \%$

$\rightarrow$ Proportion of malignancy in cases excluding 30-60 age group were $28.1 \%$

Table 5

Comparison between FNAC and histopathology report

\begin{tabular}{|l|c|c|}
\hline \multirow{2}{*}{ FNAC } & HISTOPATHOLOGY REPORT & MALIGNANT \\
\cline { 2 - 3 } & BENIGN & 7 \\
\hline BENIGN & 53 & 15 \\
\hline MALIGNANT & 5 & \\
\hline
\end{tabular}

Table 6

Comparison between USG and histopathology report

\begin{tabular}{|l|c|c|}
\hline \multirow{2}{*}{ USG } & \multicolumn{2}{|c|}{ HISTOPATHOLOGY REPORT } \\
\cline { 2 - 3 } & BENIGN & MALIGNANT \\
\hline BENIGN & 55 & 4 \\
\hline MALIGNANT & 3 & 18 \\
\hline
\end{tabular}

Table 7

INFERENCE

\begin{tabular}{|l|l|l|}
\hline & USG & FNAC \\
\hline Sensitivity & $81.8 \%$ & $68.1 \%$ \\
\hline Specificity & $94.8 \%$ & $91.3 \%$ \\
\hline Positive predictive value & $85.7 \%$ & $75 \%$ \\
\hline Negative predictive value & $93.2 \%$ & $88.3 \%$ \\
\hline
\end{tabular}

- Sensitivity of ultrasound has been demonstrated as high as $86.5 \%$ and specificity as high as $92.3 \%{ }^{109}$ which is almost similar in this study (sensitivity $81.8 \%$ and specificity of $94.8 \%$

- Sensitivity and accuracy of FNAC is as high as $95 \%$ in experienced hands .It has a positive predictive value of $90-98 \%$ and negative predictive value of $94-99 \%{ }^{107}$ in literature.

- But in this study the sensitivity and specificity of FNAC is found to be $68.1 \%$ and $91.3 \%$ respectively which is less than the values in literature.

- In our settings we found that FNAC results are usually not confirmatory.

- The higher values of sensitivity and specificity in ultrasound may be due to experienced ultrasonographist.

Table 8

SONOGRAPHIC FEATURES IN MALIGNANCY

\begin{tabular}{|l|c|c|}
\hline 1.Echotexture & No. of cases & $\%$ \\
\hline hypoechoic & 18 & 81.81 \\
\hline Isoechoic & 3 & 13.63 \\
\hline Mixed & 1 & 4.54 \\
\hline Hyperechoic & 0 & 0 \\
\hline 2.Internal architecture & & 86.36 \\
\hline Solid & 19 & 9.09 \\
\hline Solid with cystic elements & 2 & \\
\hline
\end{tabular}


Sonological And Pathological Evaluation Of Solitary Nodule Thyroid

\begin{tabular}{|l|c|c|}
\hline Predominantly cystic & 1 & 4.54 \\
\hline 3.Margin & & 54.54 \\
\hline Poorly defined & 12 & 45.45 \\
\hline Well defined & & \\
\hline 4.Halo & 15 & 68.18 \\
\hline Absent & 7 & 31.81 \\
\hline Present & & 68.18 \\
\hline 5.Vascularity & 15 & 22.72 \\
\hline Intrinsic & 5 & 9.09 \\
\hline Perinodular & 2 & \\
\hline None & & 45.45 \\
\hline 6.Calcification & 10 & 4.54 \\
\hline Microcalcification & 1 & 4.54 \\
\hline Coarse calcification & 1 & 45.45 \\
\hline Peripheral calcification & 10 & \\
\hline None & & \\
\hline 7.Shape & 17 & \\
\hline Taller than wide & 5 & 22.72 \\
\hline Ovoid to round & & \\
\hline 8.Lymphnodes & 10 & \\
\hline Present & 12 & \\
\hline Absent & & \\
\hline
\end{tabular}

\section{INFERENCE}

The most common sonographic features in malignancy are Solid internal architecture $(86.36 \%)$ followed by hypoechoic echotexture $(81.81 \%)$, then taller than wide shape $(77.27 \%) \&$ absence of halo \& intrinsic vascularity $(68.18 \%)$

\section{Discussion}

The present study is a hospital based prospective study which includes a total number of 80 cases who underwent thyroidectomy in surgery department of MES medical college, perinthalmanna during the period of October 2011-2013.

\section{INCLUSION CRITERIA}

1. Patients presenting with solitary nodule thyroid

2. Patients who underwent thyroidectomy in surgery department of MES medical college perinthalmanna during October 2011-2013

\section{EXCLUSION CRITERIA}

1. Patients having multinodular or diffuse thyroid disease thyroid

2. Patients not undergone surgery

\section{STATISTICAL ANALYSIS}

Diagnostic value of USG will be calculated by sensitivity, specificity, positive predictive value, negative predictive value \& by calculating percentage of false positive and false negative cases.

Analysis will be done on $2 \times 2$ tables for sensitivity and specificity.The statistical analysis software used is SPSS and values $\leq 0.05$ considered as significant.

Final HPR will be taken as gold standard and results of USG will be taken as positive cases.

\begin{tabular}{|l|c|c|}
\hline \multirow{2}{*}{ Test } & \multicolumn{2}{|c|}{ HPR } \\
\cline { 2 - 3 } & benign & malignant \\
\hline benign & TN & FN \\
\hline malignant & FP & TP \\
\hline
\end{tabular}

- $\quad$ Sensitivity $=\mathrm{TP} / \mathrm{TP}+\mathrm{TN}$

- $\quad$ Specificity $=\mathrm{TN} / \mathrm{TN}+\mathrm{FP}$

- Positive predictive value $=\mathrm{TP} / \mathrm{TP}+\mathrm{FP}$

- $\quad$ Negative predictive value $=\mathrm{TN} / \mathrm{TN}+\mathrm{FN}$

TP- True positive

TN- True negative

FP-False Positive

FN-False negative

\section{AETIOLOGICAL FACTORS}


Family history was present in only one case and that was a case of papillary carcinoma thyroid .History of irradiation was not detected in any case

\section{SEX DISTRIBUTION}

Almost all thyroid disorders were more common in females .In total the ratio is found to be 1:5.7 which is almost similar to values in the literature ie. $1: 8^{[6]}$

\section{AGE DISTRIBUTION}

Most of the thyroid disorders were found to be clustered within the middle age group.96.25\% of the cases belong to 20-60 age group.It is in accordance with the literature ${ }^{[7]}$

\section{PROPORTION OF VARIOUS BENIGN DISORDERS}

Among various benign diseases of thyroid,colloid nodule was the commonest ( $58.62 \%)$.Thyroiditis was second (32.75\%).Proportion of follicular adenoma was $8.62 \%$

\section{AGE AND SEX PREDILECTION FOR VARIOUS BENIGN DISORDERS}

For colloid nodule male:female ratio is found to be 1:4.67 which is in accordance to the values in literature $(1: 6)^{96}$.Thyroiditis, follicular adenoma were found only in females as in literature.

\section{PROPORTION OF VARIOUS MALIGNANCIES THYROID}

Coming to proportion of various malignant diseases, that of papillary carcinoma was $68.1 \%$,follicular carcinoma thyroid was $31.8 \%$ and that of other malignancies were $0 \%$ while the values given in literature are papillary carcinoma thyroid-60-80\%,follicular carcinoma thyroid-10-17\%.

\section{AGE AND SEX PREDILECTION FOR MALIGNANCY THYROID}

Male :female ratio for malignancies was found to be 1:2.6 which is almost similar to ratio given in the literature ie, $1: 3^{[8]}$.

\section{ROLE OF FNAC}

Sensitivity of FNAC was $68.1 \%$ and specificity was $91.3 \%$.Positive predictive value of FNAC was $75 \%$ and negative predictive value of FNAC was $88.3 \%{ }^{[9]}$

\section{ROLE OF USG}

Sensitivity of USG was $81.8 \%$ and specificity was $94.8 \%$. Positive predictive value was $85.7 \%$ and negative predictive value was $93.2 \%$ [10]

Thyroid malignancy is relatively rare and is diagnosed in approximately 25,000 patients per year in the United States[11]. Although less than $7 \%$ of thyroid nodules are malignant ${ }^{[12]}$, it is critical that they be accurately identified. The imaging modality of choice for the investigation of thyroid nodules is high-resolution US. US is commonly misperceived as unhelpful in distinguishing between benign and malignant thyroid nodules. Although individual US features may be of limited value, when multiple signs of thyroid malignancy appear in combination it is possible to make an accurate prediction.

\section{US Features Suggestive of Malignancy 1)Calcifications}

Thyroid calcifications may occur in both benign and malignant disease. It can be classified as microcalcification, coarse calcification, or peripheral calcification. Thyroid microcalcifications are psammoma bodies, which are 10-100- $\mu \mathrm{m}$ round laminar crystalline calcific deposits They are one of the most specific features of thyroid malignancy, Microcalcifications are found in $29 \%-59 \%$ of all primary thyroid

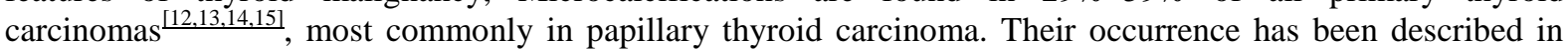
follicular and anaplastic thyroid carcinomas as well as in benign conditions such as follicular adenoma and Hashimoto thyroiditis ${ }^{[16]}$. At US, microcalcifications appear as punctate hyperechoic foci without acoustic shadowing. 2) Halo

In this study microcalcifications are found in $45.5 \%$ of malignant cases and only in one benign case

The halo or hypo-echoic rim around a thyroid nodule is produced by a pseudo-capsule of fibrous connective tissue, a compressed thyroid parenchyma, and chronic inflammatory infiltrates ${ }^{[17]}$ In this study it is found that about $94 \%$ of benign cases and $31.81 \%$ of malignant cases have presence of halo. A completely uniform halo around a nodule is highly suggestive of benignity, with a specificity of $95 \%{ }^{[18]}$. However, a halo is absent at US in more than half of all benign thyroid nodules ${ }^{[17,19]}$. Moreover, $10 \%-24 \%$ of papillary thyroid carcinomas have either a complete or an incomplete halo ${ }^{[14,19-20]}$.so absence of halo is not a typical predictor of malignancy

\section{3) Margin}

A thyroid nodule is considered ill defined when more than $50 \%$ of its border is not clearly demarcated. An ill-defined and irregular margin in a thyroid tumor suggests malignant infiltration of adjacent thyroid 
parenchyma with no pseudocapsule formation. The reported sensitivity of ill-defined margins and irregular margins, however, ranges widely $(53 \%-89 \%)^{[21]}$ The specificity of ill-defined margins is variable, with $15 \%$ $59 \%$ of benign nodules having poorly defined margins with macro- or microlobulations ${ }^{[21,22]}$. Therefore, unless frank invasion beyond the capsule is demonstrated, the US appearance of the nodule margins alone is an unreliable basis for determining malignancy or benignity.in this study we found irregular margins in 5 benign and 6 malignant cases. Irregular margin is seen in $54.54 \%$ of malignant cases in this study

\section{4) Shape}

The shape of a thyroid nodule is a potentially useful US feature that has not been extensively described in the literature. Kim et $\mathrm{al}^{[13]}$ found that a solid thyroid nodule that is taller than it is wide (ie, greater in its anteroposterior dimension than its transverse dimension) has a $93 \%$ specificity for malignancy. This appearance is thought to be due to a centrifugal tendency in tumor growth, which does not necessarily occur at a uniform rate in all dimensions.in this study it is found that $77.27 \%$ of cases showing this feature

\section{5) Vascularity}

Vascular flow within a thyroid nodule can be detected with color or power Doppler US. The most common pattern of vascularity in thyroid malignancy is marked intrinsic hypervascularity, which is defined as flow in the central part of the tumor that is greater than that in the surrounding thyroid parenchyma.. This occurs in $69 \%-74 \%$ of all thyroid malignancies ${ }^{[12,14]}$. However, it is not a specific sign of thyroid malignancy by itself. In this study intrinsic vascularity is seen in $68.18 \%$ of malignant cases. Frates et al ${ }^{[22]}$ showed that more than $50 \%$ of hypervascular solid thyroid lesions were benign. In this study intrinsic vascularity is seen in $32.35 \%$ of benign casesPerinodular flow is defined as the presence of vascularity around at least $25 \%$ of the circumference of a nodule. This flow pattern is more characteristic of benign thyroid lesions but also has been found in $22 \%$ of thyroid malignancies ${ }^{[14]}$. In contrast, complete avascularity is a more useful sign: Chan et al ${ }^{[14]}$ reported that all papillary thyroid carcinomas in their study had some intrinsic blood flow, and they concluded that a completely avascular nodule is very unlikely to be malignant.complete avascularity and perinodular flow pattern is seen in only $1.81 \%$ of malignancies.

\section{6)Hypoechoic Solid Nodule}

Malignant nodules, both carcinoma and lymphoma, typically appear solid and hypoechoic when compared with normal thyroid parenchyma. The combination of these two US features has a sensitivity of $87 \%$ for the detection of thyroid malignancy ${ }^{[12]}$. Marked hypoechogenicity is very suggestive of malignancy. Solid internal echotexture is seen in $86.36 \%$ of malignant cases and hypoechogenicity is seen in $81.81 \%$ of cases

Although a single feature in ultrasound is not helpful in predicting malignancy, a combination of solid, hypoechoic nodule with poorly defined margins and intrinsic vascularity and microcalcifications can almost accurately predict malignancy suggestive of benignity, with a specificity of $95 \%{ }^{[18]}$. However, a halo is absent at US in more than half of all benign thyroid nodules ${ }^{[17,19]}$. Moreover, $10 \%-24 \%$ of papillary thyroid carcinomas have either a complete or an incomplete halo ${ }^{[14,18-20]}$.so absence of halo is not a typical predictor of malignancy

Although a single feature in ultrasound is not helpful in predicting malignancy, a combination of solid, hypoechoic nodule with poorly defined margins and intrinsic vascularity and microcalcifications can almost accurately predict malignancy.

\section{Conclusion}

1. All thyroid disorders were common in females, the commonest age group being 20-60 years.

2. Nodular colloid goiter formed majority of thyroid diseases $(58.62 \%)$ and one third were due to thyroiditis.Rest of the cases of were due to follicular adenoma (8.62\%)

3. thyroid malignancies were more common in females and middle age group .

4. sensitivity and specificity of USG neck was high when compared to FNAC and is found as a valuable tool in diagnosis of thyroid diseases.

5. USG features specific for malignancy in decreasing order of frequency include
a) Solid internal architecture $(86.36 \%)$
b) Hypoechoic echotexture $(81.81 \%)$
c) Intrinsic vascularity $(68.18 \%)$
d) Poorly defined margins(54.545)
e) Microcalcification(45.45\%) 


\section{References}

[1]. The spectrum of thyroid disease in a community ; The whick-harm survey.clin Endocrinol(oxf)7:481-490

[2]. Risk of malignancy in non-palpable thyroid nodule;predictive value of ultrasound and color Doppler features.J clin Endocrinol metab 2002;87(5):1941-1946.

[3]. Ultra sound guided fine needle aspirating biopsy, aspiration biopsy of thyroid nodules, comparison in efficacy according to nodule size.Thyroid 2009 jan;19(1)27-31 American thyroid association guidelines 2009 Koike et al 2001

[4]. Belfiore A, La Rosa GL, La Porta GA et al: Cancer risk in patients with cold thyroid nodules. Relevance of iodine intake, sex, age and multinodularity. Am J Med 93:363-369, 1992.

[5]. Zygmunk HK: The thyroid gland and the thyroglossal tract in Russell RCG, Williams NS, Bulstrode CJK(eds): Bailey and Love's Short Practice of Surgery, $23^{\text {rd }}$ edn. London: Arnold, 2000, p 713.

[6]. John BH: Thyroid in Courtney MT, Daniel RB, Mark B and et al. (eds): Sabiston Text book of Surgery, 17 ${ }^{\text {th }}$ edn. Philadelphia: saunders, 2004, p 961.

[7]. Khafagi F, Wright G, Castles H et al. screening for thyroid malignancy: the role of fine needle biopsy. Med J Aust, 1988:149:302303, 306-307.

[8]. Okamota Yamashita T, Haraswara A et al: Test performances of the diagnostic procedures in evaluating thyroid nodules: Physical examinations, ultrasonography, and fine needle aspiration cytology, Endocr J 1994:41:243-247.

[9]. FratesMC, Benson CB, Charboneau JW, et al. Management of thyroid nodules detected at US: Society of Radiologists in Ultrasound consensus conference statement. Radiology2005; 237(3): 794-800.

[10]. PapiniE, Guglielmi R, Bianchini A, et al. Risk of malignancy in nonpalpable thyroid nodules: predictive value of ultrasound and color-Doppler features. J Clin Endocrinol Metab2002; 87(5): 1941-1946.

[11]. KimEK, Park CS, Chung WY, et al. New sonographic criteria for recommending fine-needle aspiration biopsy of nonpalpable solid nodules of the thyroid. AJR Am J Roentgenol2002; 178(3): 687-691

[12]. ChanBK, Desser TS, McDougall IR, Weigel RJ, Jeffrey RB Jr. Common and uncommon sonographic features of papillary thyroid carcinoma. J Ultrasound Med2003; 22(10): 1083-1090.

[13]. KlinckGH, Winship T. Psammoma bodies and thyroid cancer. Cancer1959; 12(4): 656-662

[14]. TakiS, Terahata S, Yamashita R, et al. Thyroid calcifications: sonographic patterns and incidence of cancer. Clin Imaging2004; 28(5):368-371.

[15]. PropperRA, Skolnick ML, Weinstein BJ, Dekker A. The nonspecificity of the thyroid halo sign. J Clin Ultrasound1980; 8(2): 129132

[16]. LuC, Chang TC, Hsiao YL, Kuo MS. Ultrasonographic findings of papillary thyroid carcinoma and their relation to pathologic changes. J Formos Med Assoc 1994; 93(11-12): 933-938

[17]. WattersDA, Ahuja AT, Evans RM, et al. Role of ultrasound in the management of thyroid nodules. Am J Surg 1992; 164(6): 654657

[18]. HayashiN, Tamaki N, Yamamoto K, et al. Real-time ultrasonography of thyroid nodules. Acta Radiol Diagn (Stockh)1986; 27(4): 403-408.

[19]. WienkeJR, Chong WK, Fielding JR, Zou KH, Mittelstaedt CA. Sonographic features of benign thyroid nodules: interobserver reliability and overlap with malignancy. J Ultrasound Med2003; 22(10): 1027-1031.

[20]. FratesMC, Benson CB, Doubilet PM, Cibas ES, Marqusee E. Can color Doppler sonography aid in the prediction of malignancy of thyroid nodules? J Ultrasound Med2003; 22(2): 127-131 